\title{
Analysis of Standard Gradient Descent with GD Momentum and Adaptive LR for SPR Prediction
}

\author{
Anjar Wanto ${ }^{1}$, Sundari Retno Andani ${ }^{2}$, Poningsih Poningsih ${ }^{2}$, Rafiqa Dewi ${ }^{2}$, Muhammad Ridwan \\ Lubis $^{2}$, Widodo Saputra ${ }^{2}$, Ika Okta Kirana ${ }^{1}$ \\ ${ }^{1}$ STIKOM Tunas Bangsa, Sudirman street Blok A Number 1, 2, 3 Pematangsiantar, North Sumatra - Indonesia \\ ${ }^{2}$ AMIK Tunas Bangsa, Sudirman street Blok A Number 1, 2, 3 Pematangsiantar, North Sumatra - Indonesia \\ \{anjarwanto@amiktunasbangsa.ac.id,sundari.ra@amiktunasbangsa.ac.id,poningsih@amiktunasbangsa.ac.id, \\ rafiqa.atb@gmail.com,m.ridwan@amiktunasbangsa.ac.id,widodo@amiktunasbangsa.ac.id, \\ ikaoktakirana@stikomtb.ac.id\}
}

Keywords: Analysis, ANN, GD, GDMALR, SPR.

\begin{abstract}
Gradient Descent (GD) is used to find the local minimum value, its purpose is to find variables on the error function so that a function can model the data with minimum error. Therefore, the purpose of this research is to see how much iteration is needed and how big is the accuracy level in predicting the data when using Gradient Descent (GD) Standard and GD With Momentum and Adaptive Learning Rate (GDMALR) functions. In this study, the data to be processed using the gradient descent function is the data of School Participation Rate (SPR) in Indonesia aged 19-24 years, which began in 2011 to 2017. The reason for selection This age range is one of the factors that determine success education in a country, especially Indonesia. SPR is known as one of the indicators of successful development of education services in an area of either Province, Regency or City in Indonesia. The higher the value of SPR, then the area is considered successful in providing access to education services. SPR data are taken from Indonesian Central Bureau of Statistics. This study uses 3 models of network architecture, namely: 5-5-1, 5-15-1 and 5-25-1. From 3 models, the best model is 5-5-1 with epoch 6202 iteration, 94\% accuracy and MSE 0.0008658637 . This model is then used to predict SPR in Indonesia for the next 3 years $(2018-2020)$. These results will be expected to help the Indonesian government to further improve the scholarship and improve the quality of education in the future.
\end{abstract}

\section{INTRODUCTION}

School Participation Rate (SPR) is the proportion of all school-aged children in a certain age group of people with appropriate age groups. SPR is known as one of the indicators of successful development of education services in an area of either Province, Regency or City in Indonesia. The higher the value of APS, then the area is considered successful in providing access to education services. A high APS indicates greater opportunities for access to education in general. In the age group where these opportunities occur can be seen from the magnitude of SPR in each age group. School Participation Rate is a measure of the absorptive capacity of educational institutions towards school-age population. SPR is a basic indicator used to view population access to educational facilities, especially for school-aged residents in a region. The higher the School Participation Rate the greater the number of people who have the opportunity to receive education.

Therefore, predicting the value of RPS for subsequent years is very important to do, as a barometer of the government in determining related policies in the field of education. One way to measure the value of RPS with the Neural Network using Gradient Descent function. Gradient Descent is usually used to find the local minimum value that can be generated from a parametric function, the purpose is to find variables on the error function so that a function can model the data with minimum error. The gradient descent has a linear complexity with respect to data expansion and is also easy to compute in parallel (using GPUs). This 
characteristic allows a model of a large enough neural network to be trained with millions of training data. However, the use of standard gradient descent functions in processing the data to be predicted sometimes takes a long time. So it takes another faster function to process the data, without reducing the quality of the results to be determined. The function is Gradient Descent With Momentum \& Adaptive Learning Rate (GDMALR). This function is able to speed prediction without unduly affecting the quality of the results when using a standard gradient descent.

In the previous study, Friedman proposed a gradient descent (GD) algorithm (Friedman, Hastie, et al. 2000) (Friedman. 2001) in which each calculation reduces the residual of the last model base, and a new ensemble model is established in the direction of the reduced residual. This ultimately produces a forward distribution additive model by continuous iteration, which can optimize the minimization of the loss function. A series of efficient algorithms have been obtained (Friedman, et al. 2004) (Rosset, et al. 2004) (Friedman. 2002) (Zhang, et al. 2011) (Zhang, Liu, et al. 2013).

The contribution of this research are:

- As an input for academics to use the gradient descent With Momentum \& Adaptive Learning Rate (GDMALR) training function to predict because training with this method is proven to be fast without unduly influencing the quality of the results obtained.

- As an input for the government to be able to make appropriate policies to advance education by pressing the numbers of people who do not go to school or drop out of school.

\section{RUDIMENTARY}

\subsection{Artificial Intelligence}

Artificial Intelligence is one area that is quite reliable in solving problems such as prediction (forecasting) (Wanto, Zarlis, et al. 2017). AI is a very important discipline and it includes a number of well recognized and mature areas including Neural Network (Chiroma, et al. 2014) (Hakim, et al. 2014) (Lasisi, et al. 2014). Artificial Intelligence (AI) is a general term that implies the use of a computer to model intelligent behavior with minimal human intervention. AI is generally accepted as having started with the invention of robots. The term derives from the Czech word robota, meaning biosynthetic machines used as forced labor (Hamet and Tremblay. 2017). AI is a field of study based on the premise that intelligent thought can be regarded as a form of computation - one that can be formalized and ultimately mechanized. To achieve this, however, two major issues need to be addressed. The first issue is knowledge representation, and the second is knowledge manipulation (Sumijan, et al. 2016).

\subsection{Artificial Neural Networks (NN)}

Artificial Neural Network (ANN) is one of the studies of Artificial Intelligence and is a new computing technology in the field of computer science study. Neural networks mostly used for problem-solving in pattern recognition, data analysis, control and clustering (J. Adnan et al. 2017). Initially ANN were developed in the field of artificial intelligence and were first introduced for image recognition. The central concept was inspired by knowledge of the nervous system, especially the human brain with its closely connected neurons (Ehret, et al. 2015). Artificial neural network (ANN) is one of the methods that is suitable to deal with the internal relations of complex model because of its highly nonlinear, large amounts of data parallel processing, high robustness, and fault tolerance (Wang, et al. 2017) (Wanto, et al. 2017).

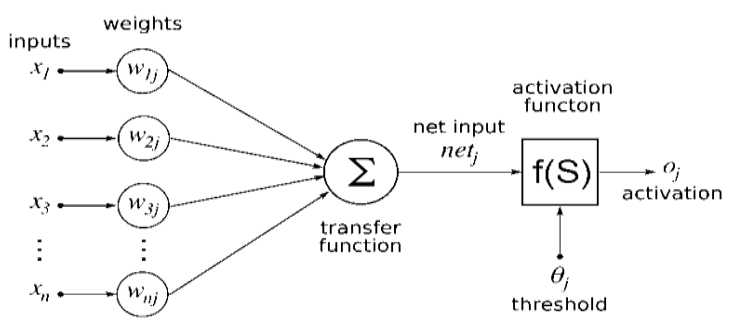

Figure 1: Artificial Neural Networks

The schematic representation of artificial neural network. It consists of: (1) input layer (independent variables), (2) hidden layers and, (3) the output layer (dependent variable) (Parveen and Danish. 2017) (Siregar and Wanto. 2017)

\subsection{Architecture of Backpropagation}

The back-propagation learning algorithm (BPLA) has become famous learning algorithms among ANNs. In the learning process, to reduce the inaccuracy of ANNs, BPLAs use the gradient-decent search method to adjust the connection weights. The structure of a back-propagation ANN is shown in Figure 2. The output of each neuron is the aggregation of the numbers of neurons of the 
previous level multiplied by its corresponding weights. The input values are converted into output signals with the calculations of activation functions. Backpropagation ANNs have been widely and successfully applied in diverse applications, such as pattern recognition, location selection and performance evaluations (Che, et al. 2011).

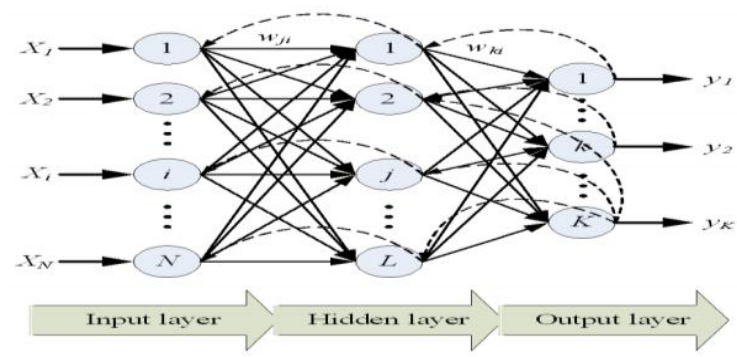

Figure 2: Back-propagation ANN

There are several algorithms that can be used to create an artificial neural network, but the Back propagation was chosen because it is probably the easiest to implement, while preserving efficiency of the network. Backward Propagation Artificial Neural Network (ANN) use more than one input layers (usually 3). Each of these layers must be either of the following:

a. Input Layer : This layer holds the input for the network.

b. Output Layer : This layer holds the output data, usually an identifier for the input.

c. Hidden Layer : This layer comes between the input layer and the output layer. They serve as a propagation point for sending data from the previous layer to the next layer (Choudhary, et al. 2013).

\subsection{Backpropagation Neural Network}

Backpropagation (BP) algorithm was used to develop the ANN model (Antwi, et al. 2017). The typical topology of BPANN (Backpropagation Artificial Neural Network) involves three layers: input layer,where the data are introduced to the network; hidden layer, where the data are processed; and output layer,where the results of the given input are produced (Huang and $\mathrm{Wu}$. 2017). A backpropagation algorithm was used for training. It is a convenient and simple iterative algorithm that usually performs well, even with complex data. Unlike other learning algorithms (like Bayesian learning) it has good computational properties when dealing with largescale data (Ehret, et al. 2015). Backpropagation training method involves feedforward of the input training pattern, calculation and backpropagation of error, and adjustment of the weights in synapses (Tarigan, et al. 2017).

\subsection{Descent Gradient}

Descent gradient methods are the most frequently used algorithms for computing regularizers of inverse problems. They are either directly applied to the discrepancy term, which measures the difference between operator evaluation and data or to a regularzied version incorporating suitable penalty terms. In its basic form, gradient descent methods converge slowly (Muoi, et al. 2015). Despite its simplicity, gradient descent algorithm suffers from variety of drawbacks. On of the main problem relates to the learning rate parameter: if it is chosen to be too big the estimate will dangle around the optimal point, not approaching it. On the other hand, if it is too small, convergence might take vast amount of iterations and we won't approach optimum for iterations (Senov and Granichin 2017).

Gradient descent backpropogation artificial neural network has been successively used for prediction of school enrollment rates by considerable number of researchers. Its performance can be improved by using variable learning rate during the training process i.e instead of using constant learning rate; adaptive learning rate can be employed. The affect of using variable learning rate will be larger step size and stable learning. In this study Gradient Descent With Momentum \& Adaptive LR is used for prediction of school participation rate. The result is compared with standard gradient descent algorithm, Gradient Descent With Momentum \& Adaptive LR is better (Singh, Verma, and Thoke 2015)..

\section{RESULT AND DISCUSSION}

\subsection{Data Used}

The data used in this study is data on School Participation Rate by province in Indonesia aged 19. 24 years. Sources of data were taken from Indonesian Central Bureau of Statistics in 20112017.

Table 1: Dataset School Participation Rate in Indonesia

School Participation Rate (aged 19-24 years)

\begin{tabular}{|l|l|l|l|l|l|}
\hline \multirow{2}{*}{ Province } & \multicolumn{5}{|c|}{ Year (In Tens of Thousands) } \\
\cline { 2 - 7 } & 2011 & 2012 & $\ldots$ & 2016 & 2017 \\
\hline
\end{tabular}




\begin{tabular}{|c|c|c|c|c|c|}
\hline \multicolumn{6}{|c|}{ School Participation Rate (aged 19-24 years) } \\
\hline \multirow{2}{*}{ Province } & \multicolumn{5}{|c|}{ Year (In Tens of Thousands) } \\
\hline & 2011 & 2012 & $\ldots$ & 2016 & 2017 \\
\hline Aceh & 27,68 & 28,55 & $\ldots$ & 33,94 & 34,28 \\
\hline Sumatera Utara & 16,94 & 17,27 &.. & 26,62 & 26,80 \\
\hline Sumatera Barat & 23,95 & 27,55 & $\ldots$ & 34,71 & 35,45 \\
\hline Riau & 15,34 & 15,81 & $\ldots$ & 26,18 & 27,28 \\
\hline Jambi & 15,64 & 15,22 & $\ldots$ & 23,86 & 24,12 \\
\hline Sumatera Selatan & 12,75 & 13,91 & $\ldots$ & 18,07 & 19,17 \\
\hline Bengkulu & 17,02 & 19,64 & $\ldots$ & 28,93 & 29,90 \\
\hline Lampung & 10,39 & 11,90 & $\ldots$ & 19,72 & 20,96 \\
\hline Kep. Bangka Belitung & 8,63 & 9,30 & $\ldots$ & 13,81 & 14,99 \\
\hline Kep. Riau & 9,67 & 10,14 & $\ldots$ & 18,58 & 19,13 \\
\hline DKI Jakarta & 17,83 & 18,02 & $\ldots$ & 23,06 & 24,60 \\
\hline Jawa Barat & 11,15 & 12,25 & $\ldots$ & 20,37 & 21,50 \\
\hline Jawa Tengah & 11,51 & 11,83 & $\ldots$ & 21,59 & 22,13 \\
\hline DI Yogyakarta & 44,17 & 44,69 & $\ldots$ & 49,95 & 51,33 \\
\hline Jawa Timur & 12,69 & 14,59 & $\ldots$ & 22,67 & 23,34 \\
\hline Banten & 13,56 & 15,97 & $\ldots$ & 20,74 & 21,33 \\
\hline Bali & 18,93 & 18,99 & $\ldots$ & 25,36 & 26,56 \\
\hline NTB & 16,99 & 17,82 & $\ldots$ & 27,79 & 28,52 \\
\hline NTT & 17,40 & 17,92 & $\ldots$ & 26,75 & 27,80 \\
\hline Kalimantan Barat & 11,94 & 14,17 & $\ldots$ & 24,75 & 25,80 \\
\hline Kalimantan Tengah & 13,05 & 14,04 & $\ldots$ & 22,72 & 24,15 \\
\hline Kalimantan Selatan & 13,62 & 16,48 & $\ldots$ & 21,89 & 23,53 \\
\hline Kalimantan Timur & 16,92 & 20,33 & $\ldots$ & 28,88 & 30,04 \\
\hline Kalimantan Utara & 0,00 & 0,00 & $\ldots$ & 19,07 & 20,72 \\
\hline Sulawesi Utara & 15,16 & 16,12 & $\ldots$ & 22,82 & 24,22 \\
\hline Sulawesi Tengah & 16,72 & 16,74 & $\ldots$ & 25,57 & 26,31 \\
\hline Sulawesi Selatan & 21,46 & 23,17 & $\ldots$ & 31,48 & 32,16 \\
\hline Sulawesi Tenggara & 21,48 & 23,62 & $\ldots$ & 29,31 & 30,03 \\
\hline Gorontalo & 19,85 & 20,46 & $\ldots$ & 28,98 & 29,21 \\
\hline Sulawesi Barat & 13,03 & 14,65 & $\ldots$ & 22,36 & 23,49 \\
\hline Maluku & 26,71 & 28,98 & $\ldots$ & 37,51 & 38,20 \\
\hline Maluku Utara & 19,33 & 21,79 & $\ldots$ & 31,75 & 32,10 \\
\hline Papua Barat & 16,46 & 20,03 & $\ldots$ & 31,45 & 31,92 \\
\hline Papua & 12,81 & 13,86 & $\ldots$ & 23,75 & 24,57 \\
\hline Indonesia & 14,82 & 16,05 & $\ldots$ & 23,93 & 24,77 \\
\hline
\end{tabular}

Based on table 1. It can be pointed out that the 20112015 School Participation Figures dataset is used for training with targets for 2016, while the 2012-2016 dataset is used for testing targeted by 2017 .

\subsection{Normalization Data}

The data will be normalized using the following formula.

$$
x^{\prime}=\frac{0.8(x-a)}{b-a}+0.1
$$

Table 2: Normalization of training data

\begin{tabular}{|l|c|c|c|c|c|}
\hline \multirow{2}{*}{\multicolumn{1}{|c|}{ Province }} & \multicolumn{5}{|c|}{ Years } \\
\cline { 2 - 6 } & 2011 & 2012 & $\ldots$ & 2015 & Target \\
\hline Aceh & 0,5433 & 0,5573 & $\ldots$ & 0,6296 & 0,6436 \\
\hline Sumatera Utara & 0,3713 & 0,3766 & $\ldots$ & 0,5030 & 0,5263 \\
\hline Sumatera Barat & 0,4836 & 0,5412 & $\ldots$ & 0,6306 & 0,6559 \\
\hline Riau & 0,3457 & 0,3532 & $\ldots$ & 0,4980 & 0,5193 \\
\hline Jambi & 0,3505 & 0,3438 & $\ldots$ & 0,4559 & 0,4821 \\
\hline Sumatera Selatan & 0,3042 & 0,3228 & $\ldots$ & 0,3723 & 0,3894 \\
\hline Bengkulu & 0,3726 & 0,4146 & $\ldots$ & 0,5544 & 0,5633 \\
\hline Lampung & 0,2664 & 0,2906 & $\ldots$ & 0,4013 & 0,4158 \\
\hline Kep. Bangka Belitung & 0,2382 & 0,2489 & $\ldots$ & 0,3039 & 0,3212 \\
\hline Kep. Riau & 0,2549 & 0,2624 & $\ldots$ & 0,3833 & 0,3976 \\
\hline DKI Jakarta & 0,3856 & 0,3886 & $\ldots$ & 0,4637 & 0,4693 \\
\hline Jawa Barat & 0,2786 & 0,2962 & $\ldots$ & 0,4107 & 0,4262 \\
\hline Jawa Tengah & 0,2843 & 0,2895 & $\ldots$ & 0,4294 & 0,4458 \\
\hline DI Yogyakarta & 0,8074 & 0,8158 & $\ldots$ & 0,8875 & 0,9000 \\
\hline Jawa Timur & 0,3032 & 0,3337 & $\ldots$ & 0,4516 & 0,4631 \\
\hline Banten & 0,3172 & 0,3558 & $\ldots$ & 0,4152 & 0,4322 \\
\hline Bali & 0,4032 & 0,4041 & $\ldots$ & 0,4804 & 0,5062 \\
\hline NTB & 0,3721 & 0,3854 & $\ldots$ & 0,5299 & 0,5451 \\
\hline NTT & 0,3787 & 0,3870 & $\ldots$ & 0,5251 & 0,5284 \\
\hline Kalimantan Barat & 0,2912 & 0,3269 & $\ldots$ & 0,4735 & 0,4964 \\
\hline Kalimantan Tengah & 0,3090 & 0,3249 & $\ldots$ & 0,4599 & 0,4639 \\
\hline Kalimantan Selatan & 0,3181 & 0,3639 & $\ldots$ & 0,4288 & 0,4506 \\
\hline Kalimantan Timur & 0,3710 & 0,4256 & $\ldots$ & 0,5412 & 0,5625 \\
\hline Kalimantan Utara & 0,1000 & 0,1000 & $\ldots$ & 0,3862 & 0,4054 \\
\hline Sulawesi Utara & 0,3428 & 0,3582 & $\ldots$ & 0,4413 & 0,4655 \\
\hline Sulawesi Tengah & 0,3678 & 0,3681 & $\ldots$ & 0,5025 & 0,5095 \\
\hline Sulawesi Selatan & 0,4437 & 0,4711 & $\ldots$ & 0,5907 & 0,6042 \\
\hline Sulawesi Tenggara & 0,4440 & 0,4783 & $\ldots$ & 0,5627 & 0,5694 \\
\hline Gorontalo & & & $\ldots$ & 0,5545 & 0,5641 \\
\hline
\end{tabular}




\begin{tabular}{|l|c|c|c|c|c|}
\hline \multirow{2}{*}{\multicolumn{1}{|c|}{ Province }} & \multicolumn{5}{|c|}{ Years } \\
\cline { 2 - 6 } & 2011 & 2012 & $\ldots$ & 2015 & Target \\
\hline Sulawesi Barat & 0,3087 & 0,3346 & $\ldots$ & 0,4519 & 0,4581 \\
\hline Maluku & 0,5278 & 0,5641 & $\ldots$ & 0,6862 & 0,7008 \\
\hline Maluku Utara & 0,4096 & 0,4490 & $\ldots$ & 0,6005 & 0,6085 \\
\hline Papua Barat & 0,3636 & 0,4208 & $\ldots$ & 0,5798 & 0,6037 \\
\hline Papua & 0,3052 & 0,3220 & $\ldots$ & 0,4612 & 0,4804 \\
\hline Indonesia & 0,3374 & 0,3571 & $\ldots$ & 0,4676 & 0,4833 \\
\hline
\end{tabular}

Table 3: Normalization of testing data

\begin{tabular}{|c|c|c|c|c|c|}
\hline \multirow{2}{*}{ Province } & \multicolumn{5}{|c|}{ Years } \\
\hline & 2012 & 2013 & $\ldots$ & 2016 & Target \\
\hline Aceh & 0,5450 & 0,5548 & $\ldots$ & 0,6290 & 0,6343 \\
\hline Sumatera Utara & 0,3692 & 0,4399 & $\ldots$ & 0,5149 & 0,5177 \\
\hline Sumatera Barat & 0,5294 & 0,5778 & $\ldots$ & 0,6410 & 0,6525 \\
\hline Riau & 0,3464 & 0,4435 & $\ldots$ & 0,5080 & 0,5252 \\
\hline Jambi & 0,3372 & 0,4156 & $\ldots$ & 0,4719 & 0,4759 \\
\hline Sumatera Selatan & 0,3168 & 0,3194 & $\ldots$ & 0,3816 & 0,3988 \\
\hline Bengkulu & 0,4061 & 0,4759 & $\ldots$ & 0,5509 & 0,5660 \\
\hline Lampung & 0,2855 & 0,3523 & $\ldots$ & 0,4073 & 0,4267 \\
\hline Kep. Bangka Belitung & 0,2449 & 0,2474 & $\cdots$ & 0,3152 & 0,3336 \\
\hline Kep. Riau & 0,2580 & 0,3314 & $\ldots$ & 0,3896 & 0,3981 \\
\hline DKI Jakarta & 0,3808 & 0,4063 & $\ldots$ & 0,4594 & 0,4834 \\
\hline Jawa Barat & 0,2909 & 0,3703 & $\cdots$ & 0,4175 & 0,4351 \\
\hline Jawa Tengah & 0,2844 & 0,3715 & $\ldots$ & 0,4365 & 0,4449 \\
\hline DI Yogyakarta & 0,7965 & 0,8147 & $\ldots$ & 0,8785 & 0,9000 \\
\hline Jawa Timur & 0,3274 & 0,4038 & $\cdots$ & 0,4533 & 0,4638 \\
\hline Banten & 0,3489 & 0,3818 & $\ldots$ & 0,4232 & 0,4324 \\
\hline Bali & 0,3960 & 0,4092 & $\ldots$ & 0,4952 & 0,5139 \\
\hline NTB & 0,3777 & 0,4529 & $\cdots$ & 0,5331 & 0,5445 \\
\hline NTT & 0,3793 & 0,4566 & $\ldots$ & 0,5169 & 0,5333 \\
\hline Kalimantan Barat & 0,3208 & 0,4003 & $\ldots$ & 0,4857 & 0,5021 \\
\hline Kalimantan Tengah & 0,3188 & 0,4100 & $\cdots$ & 0,4541 & 0,4764 \\
\hline Kalimantan Selatan & 0,3568 & 0,3642 & $\ldots$ & 0,4412 & 0,4667 \\
\hline Kalimantan Timur & 0,4169 & 0,4903 & $\ldots$ & 0,5501 & 0,5682 \\
\hline Kalimantan Utara & 0,1000 & 0,1000 & $\ldots$ & 0,3972 & 0,4229 \\
\hline Sulawesi Utara & 0,3512 & 0,3550 & $\ldots$ & 0,4557 & 0,4775 \\
\hline Sulawesi Tengah & 0,3609 & 0,4391 & $\ldots$ & 0,4985 & 0,5101 \\
\hline Sulawesi Selatan & 0,4611 & 0,5333 & $\ldots$ & 0,5906 & 0,6012 \\
\hline Sulawesi Tenggara & 0,4681 & 0,4741 & $\ldots$ & 0,5568 & 0,5680 \\
\hline
\end{tabular}

\begin{tabular}{|l|c|c|c|c|c|}
\hline \multirow{2}{*}{\multicolumn{1}{|c|}{ Province }} & \multicolumn{5}{c|}{ Years } \\
\cline { 2 - 7 } & 2012 & 2013 & $\ldots$ & 2016 & Target \\
\hline Gorontalo & 0,4189 & 0,4627 & $\ldots$ & 0,5517 & 0,5553 \\
\hline Sulawesi Barat & 0,3283 & 0,3812 & $\ldots$ & 0,4485 & 0,4661 \\
\hline Maluku & 0,5517 & 0,6268 & $\ldots$ & 0,6846 & 0,6954 \\
\hline Maluku Utara & 0,4396 & 0,5118 & $\ldots$ & 0,5948 & 0,6003 \\
\hline Papua Barat & 0,4122 & 0,4756 & $\ldots$ & 0,5902 & 0,5975 \\
\hline Papua & 0,3160 & 0,3727 & $\ldots$ & 0,4702 & 0,4829 \\
\hline Indonesia & 0,3501 & 0,4139 & $\ldots$ & 0,4730 & 0,4861 \\
\hline
\end{tabular}

\subsection{Analysis And Results}

\subsubsection{Analysis}

This study uses 3 architectural models, among others: 5-5-1, 5-15-1, 5-25-1. The training and test parameters use Target Minimum Error $=0.001$ 0.05, Maximum Epoch $=15000$ and Learning Rate $=$ 0.01 . The standard gradient descent and gradient descent with Momentum and adaptive LR functions will use the same architectural and parameter model in system processing.

a. Gradient Descent Standard
$>>$ in' ', 'traingd');
$>$ net.IW $\{1,1\}$;
$>$ net.b $\{1\}$;
$>$ net. $L W\{2,1\}$;
$>$ net.b $\{2\}$
$>$ net.trainparam.epochs $=15000$;
$>>$ net.trainparam. $L r=0.01$;
$>$ net.trainParam.goal $=0.001$;
$>$ net.trainParam.show $=1000$;
$>$ net=train(net,P,T);

net $=$ newff( $\operatorname{minmax}(P),[$ Hidden,Target],\{'tansig','purel

b. Gradient Descent with Momentum and Adaptive LR $>>$

net $=$ newff(minmax $(P),[$ Hidden,Target], \{'tansig',' purel in'\},'traingdx');

$>$ net.IW $\{1,1\}$

$>$ net.b $\{1\}$

$>$ net. $L W\{2,1\}$

$>$ net.b $\{2\}$

$>$ net.trainParam.epochs $=15000$;

$>$ net.trainParam.goal $=0.001$;

$>$ net $=\operatorname{train}($ net $, P, T)$

\subsubsection{Results}

Overall, the best results of the 3 network architecture models used are 5-5-1, with the same accuracy $94 \%$ when using standard gradient descent training functions as well as gradient descent with 
momentum and adaptive LR. The difference lies in the iteration that occurs. Iterations on the standard gradient descent of 6202 and 130 on the gradient descent with momentum and adaptive LR.

For more details can be seen in the following picture:

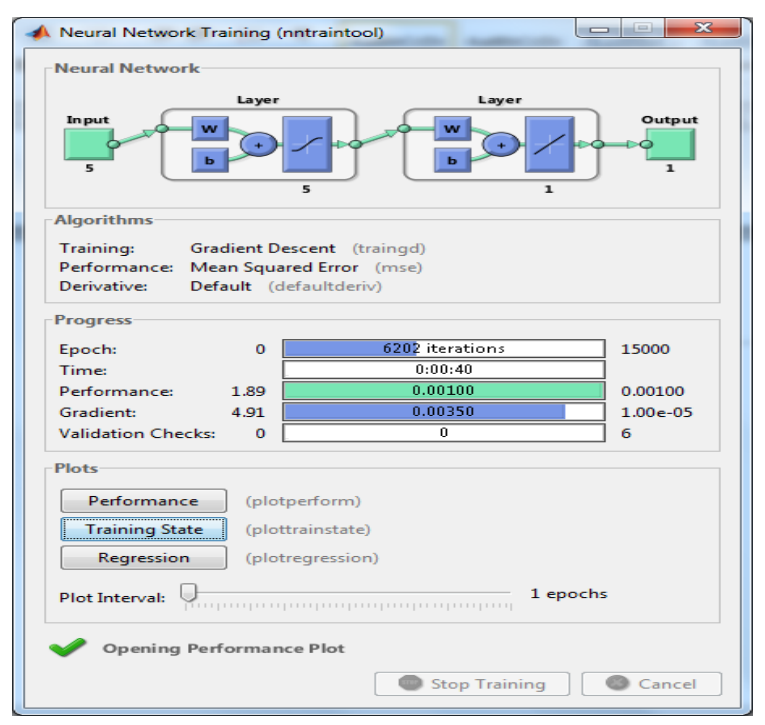

Figure 3: Training with Standard Gradient Descent

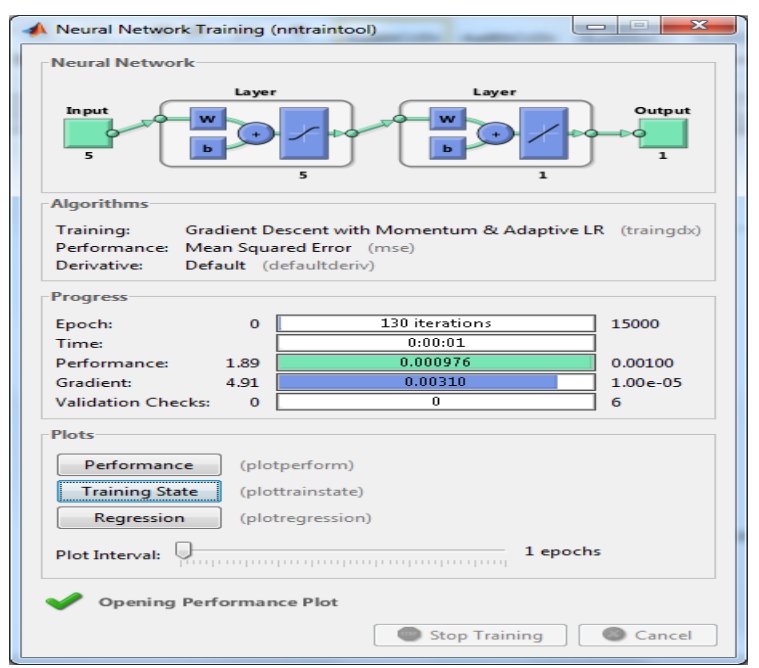

Figure 4: Training with Momentum and Adaptive LR

Comparison of standard gradient descent training functions with gradient descent with momentum and adaptive LR can be seen in the following table:

Table 4: Results with Gradient Descent

\begin{tabular}{|c|c|c|c|}
\hline \multirow{2}{*}{ Arsitektur } & \multicolumn{3}{|c|}{ Gradient Descent Standard } \\
\cline { 2 - 4 } & Iterasi & MSE & Accuracy \\
\hline $5-5-1$ & 6202 & 0,0008658637 & $94 \%$ \\
\hline
\end{tabular}

\begin{tabular}{|c|c|c|c|}
$5-15-1$ & 10633 & 0,0008604072 & $58 \%$ \\
\hline $5-25-1$ & 1407 & 0,0006577640 & $89 \%$ \\
\hline
\end{tabular}

Table 5: Results with GD Momentum and Adaptive LR

\begin{tabular}{|c|c|c|c|}
\hline \multirow{2}{*}{ Arsitektur } & \multicolumn{3}{|c|}{ With Momentum \& Adaptive LR } \\
\cline { 2 - 4 } & Iterasi & MSE & Accuracy \\
\hline $5-5-1$ & 130 & 0,0008708473 & $94 \%$ \\
\hline $5-15-1$ & 322 & 0,0008283832 & $58 \%$ \\
\hline $5-25-1$ & 96 & 0,0006339944 & $89 \%$ \\
\hline
\end{tabular}

From table 4 and 5 it can be explained that the best architectural model of 3 architecture model used is 5-5-1. Test results from the 2 training functions with architectural model 5-5-1 can be seen in the following table:

Table 6: Results of Testing Standard Gradient Descent

\begin{tabular}{|c|c|c|c|c|c|}
\hline Pattern & Target & Output & Error & SSE & Results \\
\hline Pat 1 & 0,63427 & 0,72350 & $-0,08923$ & 0,0079622679 & 1 \\
\hline Pat 2 & 0,51769 & 0,55390 & $-0,03621$ & 0,0013112032 & 1 \\
\hline Pat 3 & 0,65250 & 0,70430 & $-0,05180$ & 0,0026828868 & 1 \\
\hline Pat 4 & 0,52517 & 0,53880 & $-0,01363$ & 0,0001857642 & 1 \\
\hline Pat 5 & 0,47592 & 0,48840 & $-0,01248$ & 0,0001557376 & 1 \\
\hline Pat 6 & 0,39877 & 0,43960 & $-0,04083$ & 0,0016668727 & 1 \\
\hline Pat 7 & 0,56600 & 0,62660 & $-0,06060$ & 0,0036718406 & 1 \\
\hline Pat 8 & 0,42667 & 0,41940 & 0,00727 & 0,0000528611 & 1 \\
\hline Pat 9 & 0,33363 & 0,43370 & $-0,10007$ & 0,0100148935 & 1 \\
\hline Pat 10 & 0,39815 & 0,40090 & $-0,00275$ & 0,0000075667 & 1 \\
\hline Pat 11 & 0,48340 & 0,52360 & $-0,04020$ & 0,0001037687 & 1 \\
\hline Pat 12 & 0,43509 & 0,42490 & 0,01019 & 0,0000656993 & 1 \\
\hline Pat 13 & 0,44491 & 0,43680 & 0,00811 & 0,0049561600 & 1 \\
\hline Pat 14 & 0,90000 & 0,82960 & 0,07040 & 0,0002668688 & 0 \\
\hline Pat 15 & 0,46376 & 0,48010 & $-0,01634$ & 0,0009836270 & 1 \\
\hline Pat 16 & 0,43244 & 0,46380 & $-0,03136$ & 0,0009828879 & 1 \\
\hline Pat 17 & 0,51395 & 0,54530 & $-0,03135$ & 0,0023720411 & 1 \\
\hline Pat 18 & 0,54450 & 0,59320 & $-0,04870$ & 0,0027588874 & 1 \\
\hline Pat 19 & 0,53327 & 0,58580 & $-0,05253$ & 0,0000000384 & 1 \\
\hline Pat 20 & 0,50210 & 0,50230 & $-0,00020$ & 0,0000625985 & 1 \\
\hline Pat 21 & 0,47639 & 0,48430 & $-0,00791$ & 0,0001556228 & 1 \\
\hline Pat 22 & 0,46673 & 0,47920 & $-0,01247$ & 0,0017651555 & 1 \\
\hline Pat 23 & 0,56819 & 0,61020 & $-0,04201$ & 0,0070107230 & 1 \\
\hline Pat 24 & 0,42293 & 0,33920 & 0,08373 & 0,0000350576 & 0 \\
\hline
\end{tabular}




\begin{tabular}{|c|c|c|c|c|c|}
\hline Pattern & Target & Output & Error & SSE & Results \\
\hline Pat 25 & 0,47748 & 0,48340 & $-0,00592$ & 0,0021019840 & 1 \\
\hline Pat 26 & 0,51005 & 0,55590 & $-0,04585$ & 0,0038903472 & 1 \\
\hline Pat 27 & 0,60123 & 0,66360 & $-0,06237$ & 0,0085136806 & 1 \\
\hline Pat 28 & 0,56803 & 0,66030 & $-0,09227$ & 0,0055427517 & 1 \\
\hline Pat 29 & 0,55525 & 0,62970 & $-0,07445$ & 0,0001562076 & 1 \\
\hline Pat 30 & 0,46610 & 0,47860 & $-0,01250$ & 0,0016270465 & 1 \\
\hline Pat 31 & 0,69536 & 0,73570 & $-0,04034$ & 0,0052284141 & 1 \\
\hline Pat 32 & 0,60029 & 0,67260 & $-0,07231$ & 0,0027053678 & 1 \\
\hline Pat 33 & 0,59749 & 0,64950 & $-0,05201$ & 0,0000173559 & 1 \\
\hline Pat 34 & 0,48293 & 0,48710 & $-0,00417$ & 0,0005220749 & 1 \\
\hline Pat 35 & 0,48605 & 0,50890 & $-0,02285$ & 0,0000000000 & 1 \\
\hline
\end{tabular}

Explanation : $1=$ True $\quad 0=$ False

Table 7: Results of Testing Momentum and Adaptive LR

\begin{tabular}{|c|c|c|c|c|c|}
\hline Pattern & Target & Output & Error & SSE & Hasil \\
\hline Pat 1 & 0,63427 & 0,72650 & $-0,09223$ & 0,0085066572 & 1 \\
\hline Pat 2 & 0,51769 & 0,55030 & $-0,03261$ & 0,0010634473 & 1 \\
\hline Pat 3 & 0,65250 & 0,70750 & $-0,05500$ & 0,0030246250 & 1 \\
\hline Pat 4 & 0,52517 & 0,53620 & $-0,01103$ & 0,0001216506 & 1 \\
\hline Pat 5 & 0,47592 & 0,48480 & $-0,00888$ & 0,0000788453 & 1 \\
\hline Pat 6 & 0,39877 & 0,44280 & $-0,04403$ & 0,0019384078 & 1 \\
\hline Pat 7 & 0,56600 & 0,62580 & $-0,05980$ & 0,0035755274 & 1 \\
\hline Pat 8 & 0,42667 & 0,42340 & 0,00327 & 0,0000106966 & 1 \\
\hline Pat 9 & 0,33363 & 0,43940 & $-0,10577$ & 0,0111882321 & 1 \\
\hline Pat 10 & 0,39815 & 0,40740 & $-0,00925$ & 0,0000855767 & 1 \\
\hline Pat 11 & 0,48340 & 0,51790 & $-0,03450$ & 0,0000606326 & 1 \\
\hline Pat 12 & 0,43509 & 0,42730 & 0,00779 & 0,0000212108 & 1 \\
\hline Pat 13 & 0,44491 & 0,44030 & 0,00461 & 0,0034456900 & 1 \\
\hline Pat 14 & 0,90000 & 0,84130 & 0,05870 & 0,0002142160 & 0 \\
\hline Pat 15 & 0,46376 & 0,47840 & $-0,01464$ & 0,0007931449 & 1 \\
\hline Pat 16 & 0,43244 & 0,46060 & $-0,02816$ & 0,0006275547 & 1 \\
\hline Pat 17 & 0,51395 & 0,53900 & $-0,02505$ & 0,0022376310 & 1 \\
\hline Pat 18 & 0,54450 & 0,59180 & $-0,04730$ & 0,0025225619 & 1 \\
\hline Pat 19 & 0,53327 & 0,58350 & $-0,05023$ & 0,0000000092 & 1 \\
\hline Pat 20 & 0,50210 & 0,50220 & $-0,00010$ & 0,0000520118 & 1 \\
\hline Pat 21 & 0,47639 & 0,48360 & $-0,00721$ & 0,0000897735 & 1 \\
\hline Pat 22 & 0,46673 & 0,47620 & $-0,00947$ & 0,0015220730 & 1 \\
\hline
\end{tabular}

\begin{tabular}{|c|c|c|c|c|c|}
\hline Pattern & Target & Output & Error & SSE & Hasil \\
\hline Pat 23 & 0,56819 & 0,60720 & $-0,03901$ & 0,0071622471 & 1 \\
\hline Pat 24 & 0,42293 & 0,33830 & 0,08463 & 0,0000153738 & 0 \\
\hline Pat 25 & 0,47748 & 0,48140 & $-0,00392$ & 0,0018789970 & 1 \\
\hline Pat 26 & 0,51005 & 0,55340 & $-0,04335$ & 0,0039655543 & 1 \\
\hline Pat 27 & 0,60123 & 0,66420 & $-0,06297$ & 0,0080945304 & 1 \\
\hline Pat 28 & 0,56803 & 0,65800 & $-0,08997$ & 0,0053508726 & 1 \\
\hline Pat 29 & 0,55525 & 0,62840 & $-0,07315$ & 0,0001463690 & 1 \\
\hline Pat 30 & 0,46610 & 0,47820 & $-0,01210$ & 0,0022030505 & 1 \\
\hline Pat 31 & 0,69536 & 0,74230 & $-0,04694$ & 0,0055514083 & 1 \\
\hline Pat 32 & 0,60029 & 0,67480 & $-0,07451$ & 0,0028851025 & 1 \\
\hline Pat 33 & 0,59749 & 0,65120 & $-0,05371$ & 0,0000332473 & 1 \\
\hline Pat 34 & 0,48293 & 0,48870 & $-0,00577$ & 0,0003705224 & 1 \\
\hline Pat 35 & 0,48605 & 0,50530 & $-0,01925$ & 0,0000000000 & 1 \\
\hline
\end{tabular}

Comparison of Gradient Descent Standard With Gradient Descent With Momentum \& Adaptive LR can be seen in the following figure:

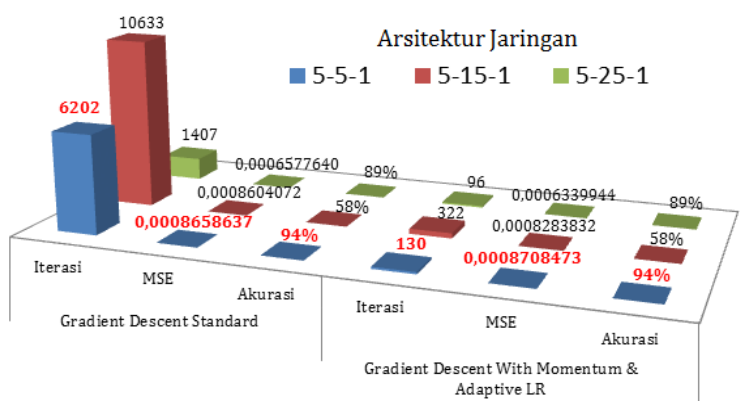

Figure 5: Graph of GD with GDMALR comparison

Comparison of prediction results using standard gradient descent and gradient descent with momentum and adaptive LR for 2018, 2019, 2020 in predicting school enrollment rates by the province in Indonesia can be seen in the following table:

Table 8: Predicted Results Using Gradient Descent

\begin{tabular}{|c|c|c|c|c|c|}
\hline \multicolumn{3}{|c|}{ Gradient Descent } & \multicolumn{3}{c|}{ GD WMALR } \\
\hline $\mathbf{2 0 1 8}$ & $\mathbf{2 0 1 9}$ & $\mathbf{2 0 2 0}$ & $\mathbf{2 0 1 8}$ & $\mathbf{2 0 1 9}$ & $\mathbf{2 0 2 0}$ \\
\hline 40,87 & 43,10 & 42,36 & 40,94 & 43,50 & 42,73 \\
\hline 33,90 & 40,88 & 42,07 & 33,72 & 40,97 & 42,31 \\
\hline 40,85 & 43,10 & 42,33 & 41,02 & 43,52 & 42,69 \\
\hline
\end{tabular}




\begin{tabular}{|l|l|l|l|l|l|}
\hline 33,39 & 41,05 & 41,97 & 33,34 & 41,14 & 42,21 \\
\hline 31,41 & 39,68 & 41,90 & 31,31 & 39,78 & 42,19 \\
\hline 30,17 & 39,11 & 42,18 & 30,13 & 39,57 & 42,52 \\
\hline 36,93 & 42,30 & 42,56 & 36,88 & 42,51 & 42,86 \\
\hline 29,20 & 38,88 & 42,27 & 29,45 & 39,23 & 42,61 \\
\hline 30,15 & 40,11 & 42,06 & 30,11 & 40,68 & 42,42 \\
\hline 28,77 & 39,03 & 42,38 & 29,09 & 39,47 & 42,72 \\
\hline 32,31 & 39,51 & 41,90 & 31,93 & 39,62 & 42,18 \\
\hline 29,30 & 39,05 & 42,24 & 29,54 & 39,34 & 42,57 \\
\hline 29,50 & 38,51 & 42,20 & 29,86 & 38,75 & 42,52 \\
\hline 46,75 & 42,84 & 42,25 & 47,04 & 43,52 & 42,66 \\
\hline 30,88 & 39,29 & 42,00 & 30,91 & 39,43 & 42,31 \\
\hline 30,69 & 39,17 & 42,11 & 30,48 & 39,44 & 42,45 \\
\hline 33,33 & 39,85 & 41,87 & 32,86 & 39,93 & 42,12 \\
\hline 35,49 & 41,60 & 42,42 & 35,44 & 41,75 & 42,67 \\
\hline 34,97 & 41,63 & 42,34 & 34,85 & 41,74 & 42,59 \\
\hline 31,80 & 39,41 & 41,83 & 31,95 & 39,50 & 42,09 \\
\hline 30,90 & 39,61 & 41,95 & 31,03 & 39,72 & 42,25 \\
\hline 31,17 & 38,51 & 41,91 & 30,93 & 38,72 & 42,23 \\
\hline 36,24 & 42,55 & 42,48 & 36,04 & 42,75 & 42,76 \\
\hline 28,35 & 38,78 & 41,35 & 28,48 & 39,40 & 41,74 \\
\hline 31,27 & 37,99 & 41,83 & 31,12 & 38,18 & 42,14 \\
\hline 33,66 & 40,82 & 42,13 & 33,56 & 40,90 & 42,37 \\
\hline 38,84 & 43,26 & 42,55 & 38,87 & 43,57 & 42,88 \\
\hline 37,50 & 42,30 & 42,60 & 37,19 & 42,52 & 42,91 \\
\hline 36,87 & 42,00 & 42,56 & 36,75 & 42,19 & 42,85 \\
\hline 30,79 & 38,61 & 41,96 & 30,86 & 38,77 & 42,27 \\
\hline 43,23 & 43,04 & 42,04 & 43,56 & 43,55 & 42,43 \\
\hline 39,57 & 42,89 & 42,50 & 39,72 & 43,21 & 42,84 \\
\hline 38,67 & 42,14 & 42,54 & 38,82 & 42,44 & 42,86 \\
\hline 31,11 & 38,30 & 41,90 & 31,33 & 38,43 & 42,19 \\
\hline 31,95 & 39,70 & 41,89 & 31,80 & 39,79 & 42,17 \\
\hline & & & & & \\
\hline 39 &
\end{tabular}

\section{CONCLUSIONS}

Based on the previous description, the following conclusions are drawn:

1. Gradient descent With Momentum \& Adaptive Learning Rate (GDMALR) training function proved faster and better used to predict than standard gradient descent,

\section{REFERENCES}

A. Ehret, D. Hochstuhl, D. Gianola, and G. Thaller. 2015. "Application of neural networks with backpropagation to genome-enabled prediction of complex traits in Holstein-Friesian and German Fleckvieh cattle." Genetics Selection Evolution 47 (1): 1-9.

A. Ehret, D. Hochstuhl, D. Gianola, and G. Thaller. 2015. "Application of neural networks with backpropagation to genome-enabled prediction of complex traits in Holstein-Friesian and German Fleckvieh cattle." Genetics Selection Evolution 47 (1): 22.

A. Lasisi, R. Ghazali and T. Herawan. 2014 "Comparative performance analysis of negative selection algorithm with immune and classification algorithms." In Recent Advances on Soft Computing and Data Mining: 441-452. Springer International Publishing.

D. Huang and Z. Wu. 2017. "Forecasting Outpatient Visits Using Empirical Mode Decomposition Coupled With Backpropagation Artificial Neural Networks Optimized by Particle Swarm Optimization.” PLoS One 12 (2): 1-18.

G. Zhang, J. Liu, H. Jia, K. Li. 2013. "The application of stochastic gradient boosting to the analysis on metabolomics data." Chin. J. Health Stat 30 (3): 323-326.

H. Chiroma, H. Abdulkareem, S. Abubakar, A.I. and T. Herawan. 2014. "Kernel functions for the support vector machine: comparing performances on crude 
oil price data." In Recent Advances on Soft Computing and Data Mining: 273-281. Springer International Publishing.

H. Zhang, Y.-t. Ding, Y. Wang, R.-z. Hu, H.-x. Gao, F.-q. Zhao. 2011. "Selecting the main factors influencing the densities of polynitroaromatic compounds via adaptive gradient boosting algorithm." Chin. J. Explos. Propellants 2: 003.

J. Adnan et al. 2017. "Multilayer Perceptron Based Activation Function On Heart Abnormality Activity." Journal of Fundamental and Applied Sciences 9: 417-432.

J. Friedman, T. Hastie, R. Tibshirani, et al. 2000. "Additive logistic regression: a statistical view of boosting (with discussion and a rejoinder by the authors)." The Annals of Statistics 28 (2): 337-407.

J. Friedman, T. Hastie, S. Rosset, R. Tibshirani, J. Zhu. 2004. "Discussion of consistency in boosting." The Annals of Statistics 32 (1), 102-107.

J.H. Friedman. 2001. "Greedy function approximation: a gradient boosting machine." The Annals of Statistics, 1189-1232.

J.H. Friedman. 2002. "Stochastic gradient boosting, Comput. Stat." The Annals of Statistics 38 (4): 367378.

J. Tarigan, Nadia, R. Diedan, and Y. Suryana. 2017. "Plate Recognition Using Backpropagation Neural Network and Genetic Algorithm." Procedia Computer Science 116: 365-372.

Muoi, Pham Quy, Dinh Nho Hao, Peter Maass, and Michael Pidcock. 2015. "Descent Gradient Methods for Nonsmooth Minimization Problems in Ill-Posed Problems." Journal of Computational and Applied Mathematics 1-26.

N. Parveen, S. Zaidi, and M. Danish. 2017. "Development of SVR-based model and comparative analysis with MLR and ANN models for predicting the sorption capacity of Cr(VI)." Process Safety Environmental Protection 107 (vi): 428-437.

N.Y. Choudhary, M.R. Patil, U. Bhadade and B.M. Chaudhari. 2013. "Signature Recognition \& Verification System Using Back Propagation Neural Network." International Jorunal of IT. " Engineering and Applied Sciences Research (IJIEASR), 2 (1): 18.

P. Antwi, et al. 2017. "Estimation of Biogas and Methane Yields in an UASB Treating Potato Starch Processing Wastewater With Backpropagation Artificial Neural Network." Journal Bioresour Technology 228: 106-115.

P. Hamet and J. Tremblay. 2017. "Artificial intelligence in medicine," Metabolism: Clinical and Experimental 69: 1-14.

Putra Siregar, Sandy and Anjar Wanto. 2017. "Analysis Accuracy of Artificial Neural Network Using Backpropagation Algorithm In Predicting Process
(Forecasting)." International Journal Of Information System \& Technology 1(1):34-42.

R.F. Hakim, E. N. Sari and T. Herawan,. 2014. "Soft Solution of Soft Set Theory for Recommendation in Decision Making." In Recent Advances on Soft Computing and Data Mining: 313-324. Springer International Publishing.

Senov, Alexander and Oleg Granichin. 2017. "Projective Approximation Based Gradient Descent Modification." IFAC-PapersOnLine 50(1):3899_ $3904 . \quad$ Retrieved (https://doi.org/10.1016/j.ifacol.2017.08.362)

Singh, Bikesh Kumar, Kesari Verma, and A. S. Thoke. 2015. "Adaptive Gradient Descent Backpropagation for Classification of Breast Tumors in Ultrasound Imaging." Procedia Computer Science 46:1601-9. Retrieved (http://dx.doi.org/10.1016/j.procs.2015.02.091)

S. Rosset, J. Zhu, T. Hastie. 2004. "Boosting as a regularized path to a maximum margin classifier." $J$. Mach. Learn. Res 5, 941-973.

Sumijan, A. P. Windarto, A. Muhammad, and Budiharjo. 2016. "Implementation of Neural Networks in Predicting the Understanding Level of Students Subject." International Journal of Software Engineering and Its Applications 10 (10): 189-204.

Wanto, Anjar, Agus Perdana Windarto, Dedy Hartama, and Iin Parlina. 2017. "Use of Binary Sigmoid Function And Linear Identity In Artificial Neural Networks For Forecasting Population Density." International Journal of Information System \& Technology 1(1):43-54.

Wanto, Anjar, Muhammad Zarlis, Sawaluddin, and Dedy Hartama. 2017. "Analysis of Artificial Neural Network Backpropagation Using Conjugate Gradient Fletcher Reeves in the Predicting Process.' Journal of Physics: Conference Series 930(1):1-7.

Z.-H. Wang, D.-Y. Gong, X. Li, G.-T. Li, and D.-H. Zhang. 2017. "Prediction of bending force in the hot strip rolling process using artificial neural network and genetic algorithm (ANN-GA)." The International Journal of Advanced Manufacturing Technology.

Z.G. Che, T.A. Chiang and Z.H. Che. 2011. "Feedforward neural networks training: A comparison between genetic algorithm and back-propagation learning algorithm." International Journal of Innovative Computing, Information and Control, 7 (10): 5839-5850. 